\title{
Germanica
}

GERMANICA $38 \mid 2006$

Voix étrangères en langue allemande

\section{Valeriu Marcu}

Ein Rumäne im literarischen Berlin

\section{Andrei Corbea-Hoisie}

\section{CpenEdition}

\section{Journals}

Édition électronique

URL : http://journals.openedition.org/germanica/380

DOI : $10.4000 /$ germanica.380

ISSN : 2107-0784

Éditeur

Université de Lille

\section{Édition imprimée}

Date de publication : 1 juin 2006

Pagination : 25-46

ISBN : 2-913857-17-5

ISSN : 0984-2632

\section{Référence électronique}

Andrei Corbea-Hoisie, « Valeriu Marcu », Germanica [Online], 38 | 2006, Online erschienen am: 19

Februar 2010, abgerufen am 06 Oktober 2020. URL : http://journals.openedition.org/germanica/380 ;

DOI : https://doi.org/10.4000/germanica.380

Ce document a été généré automatiquement le 6 octobre 2020.

(c) Tous droits réservés 


\title{
Valeriu Marcu
}

\author{
Ein Rumäne im literarischen Berlin
}

\author{
Andrei Corbea-Hoisie
}

Daß Valeriu Marcu, trotz seines rumänisch klingenden Namens, ein deutscher Schriftsteller war, steht heute außer Frage ${ }^{1}$. Mehr als ein Dutzend Bücher, die in deutscher Sprache verfaßt und von deutsch(sprachig)en Verlagen (darunter Neuer Deutscher Verlag, Hoffmann \& Campe, S.Fischer, Gustav Kiepenheuer, Paul List, Querido, Allert de Lange) veröffentlicht wurden, eine große Anzahl von Aufsätzen und Essays in verschiedenen Publikationen - darunter solche, die zwischen 1920 und 1940 für die Herausbildung der öffentlichen Meinung im deutschen Sprachraum mitbestimmend waren (z. B. Das Tagebuch, Die Weltbühne, Die Neue Rundschau, Die literarische Welt, oder im Exil Das Neue Tagebuch), - die von namhaften Kritikern (Paul Levi, Joseph Roth, Ludwig Marcuse, Willy Haas, Kurt Kersten, Axel Eggebrecht, Rudolf Olden u.a.) gezeichnetenKommentare und Rezensionen seiner Schriften, und nicht zuletzt die Übersetzungen dessen Bücher in den USA, England, Frankreich, Italien, den Niederlanden, Argentinien oder Polen zeugen von einer unverleugbaren Zugehörigkeit zum deutschen Literaturfeld der 20-er und frühen 30-er Jahre, einschließlich zur Literaturlandschaft des deutschen Exils nach $1933^{2}$, die übrigens die regelmäßige Führung seines Namens in Kürschners Deutschem Literaturkalender zwischen 1928 und 1933 institutionell nur bestätigen konnte. Die relative Vergessenheit, in die der Autor und sein Werk nach dem 2. Weltkrieg gerade in der (west- und ost-)deutschen Öffentlichkeit geraten sind ${ }^{3}$, war die Folge einer Reihe soziopolitischer Umstände, die allgemein die Reintegration der Exilliteraten in die ehemalige jetzt geteilte Heimat bedingten ${ }^{4}$. In seinem Fall trug dazu auch die Tatsache bei, daß er schon 1942 starb, zu früh, um seine Projekte für die Zeit "nach dem Sieg" verwirklichen zu können. Trotz der gelegentlichen Erwähnung Marcus in verschiedenen in den letzten Jahrzehnten erschienenen Memoiren-Bänden, wobei die Wärme, mit der ihn Ernst Jünger evoziert, nicht unbemerkt blieb, ${ }^{5}$ hat das Interesse der deutschen öffentlichkeit, besonders der Fachkreise, für sein Werk erst in den letzten Jahren langsam zugenommen, was in erster Reihe der vorsichtigen aber zugleich mutigen Initiative des Münchner Verlegers Axel Matthes zu verdanken ist ${ }^{6}$. 
Valeriu Marcu wurde als Kind einer assimilierten jüdischen Familie am 8. März 1899 in Bukarest geboren. Der Vater Samuel Marcu, Sohn eines Jassyer Schneiders, konnte durch die Bemühungen seiner älteren Geschwister an der Zürcher Technischen Hochschule studieren. Als Diplom-Ingenieur kehrte er nach Rumänien zurück, wo er die Bukarester Filiale der AEG leitete. Die Mutter Anette, geborene Krauss, hatte ein Biologiestudium abgeschlossen. Die Beziehung zwischen dem Vater und dem Sohn scheint schon frühzeitig gestört zu sein - der ständig offen ausgetragene Konflikt zwischen der positivistischen Autorität des Ingenieurs Marcu, der wohl nur auf den Anstand einer gesicherten bürgerlichen Existenz bedacht war, und dem zur Unabhängigkeit drängenden Temperament des Jungen war ein mitentscheidender Grund für dessen Entschluß, seinem eigenen Lebensweg zu folgen. Das Zusammenleben war schnell unmöglich geworden, so daß Valeriu Marcu sofort nach dem Abschluß der Grundschule in ein Wiener Internat gesteckt wurde. 1915 schickte ihn die k.u.k. Polizei schleunigst nach Bukarest zurück: Es wurde ihm vorgeworfen, Mauern mit der Losung Vive la France! beschriftet $\mathrm{zu}$ haben. Sein altersbedingtes stürmisch-romantisches Temperament sowie intensive Lektüreerlebnisse bewirkten eine frühe radikale Entscheidung, ungeachtet aller daraus erwachsenden Schwierigkeiten. In Wien, wo er vermutlich schon Joseph Roth kennenlernte, knüpfte er erste Kontakte zu linksradikalen Kreisen, in Bukarest werden diese Verbindungen intensiviert. Valeriu Marcu wird vom so-zialdemokratischen Parteiführer Cristian Rakovski unterstützt und gefördert; durch ihn lernt er 1913 Trotzki kennen, der sich als Korrespondent einer Kiewer Zeitung in Bukarest aufhielt ${ }^{7}$, um über den Balkankrieg zu berichten. Zum engsten Bekanntenkreis Marcus, der der rumänischen sozialistischen Jugendbewegung als aktiver Mitkämpfer beitritt, gehörte auch Gheorghe Cristescu, der spätere erste Generalsekretär der Kommunistischen Partei Rumäniens (1921), mit dem er in den bewegten Jahren der Neutralität Rumäniens (1914-1916) des öfteren zusammen verhaftet wurde. Als er 1938 über den von dem Stalinschen Terror verfolgten Rakovski schrieb, kam Valeriu Marcu auch auf die Atmosphäre jener Jahre zu sprechen, als er regelmäßig an den Versammlungen und Demonstrationen der Sozialisten teilnahm und zusammen mit Gleichgesinnten - u.a. auch Panait Istrati - in endlosen Diskussionen die zukünftige Revolution plante ${ }^{8}$.

Die verzweifelte Familie schickte ihn nach Zürich, wo der Vater noch viele Bekannte hatte; dort sollte Valeriu Marcu zum Abitur antreten. Mit einem Empfehungsschreiben Rakovskis ausgerüstet, nimmt jedoch Marcu Verbindung zu Kreisen der jungen Sozialisten auf, erbitterten Gegnern des Weltkrieges und der offiziellen sozialdemokratischen Führung der Zweiten Internationale. Die jungen Leute gaben eine später berühmt gewordene Zeitschrift, die Jugend-Internationale, zu deren damaligen Mitarbeitern Wladimir Lenin, Leo Trotzki, Karl Liebknecht, Grigorij Sinowjew, Karl Radek, Alexandra Kollontai u.a. zählten, unter der Leitung von Willi Münzenberg heraus, der sich später erinnern sollte: "Wir glaubten schon fixe Kerle zu sein, aber Marcu überraschte uns durch seine Lebendigkeit und durch die Schnelligkeit, mit der er Probleme erfaßte und löste"'. Am 1. Juni 1916 debütierte Valeriu Marcu in der Zeitschrift mit einem kleinen Aufsatz über die Niederwerfung der Pariser Commune und einem Bericht über die sozialistische Jugendbewegung in Rumänien ${ }^{10}$. Ein anderer Aufsatz, den er in der Nummer 6 veröffentlichte, Zur Taktik der Jugend, lenkte die Aufmerksamkeit Lenins auf sich, der sich damals gerade in Zürich befand. In einem 
posthum veröffentlichten Fragment ${ }^{11}$ erinnert sich Marcu an den Augenblick seiner Bekanntschaft mit dem bolschewistischen Führer, in dessen Nähe er sich von nun an aufhält. Marcu lernt auch Martow, Sinowjew, Radek und Paul Levi kennen, prominente Exilierte, an deren Seite er das Studium der marxistischen Literatur vertieft und sich mit den komplexen ideologischen Problemstellungen, mit der Taktik und Strategie der internationalen Arbeiterbewegung vertraut macht. Seine Begeisterung und Hingabe haben dem jungen Rumänen das Vertrauen Lenins und seiner engsten Mitarbeiter eingebracht. Er erhält einen wichtigen Auftrag: Unter dem Vorwand, seinen Wehrdienst bei der rumänischen Armee anzutreten - Rumänien stand seit dem 27. August 1916 an der Seite der Entente im Krieg -, reist Valeriu Marcu nach Paris, wo er Trotzki trifft und dann mit geheimen Botschaften an die illegalen Organisationen der Bolschewiki ${ }^{12}$ über England, Norwegen, Schweden und Finnland nach Rußland. Die zaristische Polizei verhaftet ihn aber schon in St. Petersburg und er wird an der Grenze den rumänischen Behörden übergeben. Wieder inhaftiert, wird er erst nach dem 6 . Dezember 1916 befreit, als die deutschen Truppen Bukarest besetzten. Der JugendInternationale vom 1. März 1917 kann man entnehmen, daß er sich in Bukarest aufhält ${ }^{13}$. Sechs Monate später liest man in derselben Zeitschrift folgende Notiz: "Ein Gruß aus Bukarest übermittelt allen Genossen und Kameraden unser Freund Valeriu Marcu, der dort unter den schwierigsten Verhältnissen sich bemüht, die Reste der sozial-demokratischen Partei und Jugendorganisation zusammenzuhalten" ${ }^{14}$. Obwohl die Formulierung gewiß eine Überschätzung seiner Rolle dokumentiert, muß Marcus Auftritt in Bukarest dennoch effektvoll gewesen sein, wenn man bedenkt, daß er im Ausland zu Leuten Kontakt gehabt hatte, die mit dem Sieg der russischen Revolution zu Berühmtheiten geworden waren.

4 Für seine Familie war es zweifellos ein Schock: Einerseits war sie vom Aufschub des Abiturs unangenehm überrascht, andererseits war das Leben eines engagierten sozialistischen Aktivisten in der von den deutschen und österreichisch-ungarischen Truppen besetzten rumänischen Hauptstadt voller Risiken. Valeriu Marcu hatte sich doch bald einen halb-offiziellen Status geschaffen als ständiger Mitarbeiter der Zeitschrift Scena, die der Zensur der Besatzungsbehörden unterstand. Diese Publikation, die von Adolphe de Herz und D. Karnabatt geleitet wurde und zu deren Mitarbeitern wichtige Figuren der Bukarester Literatenkreise gehörten, wurde praktisch von einer Gruppe junger, links-orientierten Schriftsteller und Journalisten kontrolliert, der es nicht selten gelang, nonkonformistisch-subversives Material in der Zeitschrift unterzubringen. Marcu veröffentlichte regelmäßig vor allem Aufsätze zur deutschen Literatur und Kultur (über Arthur Schnitzler, Hermann Bahr, Frank Wedekind, Heinrich Mann, Hermann Sudermann, Gerhart Hauptmann, Bernhard Kellermann, aber auch über Lessing, Goethe, Heine, Storm), die eine bemerkenswerte, für sein damaliges Alter zweifellos ungewöhnliche Gedanken- und Ausdrucksreife belegen. Zur selben Zeit übernimmt er, in enger Zusammenarbeit mit den in Bukarest gebliebenen sozialistischen Führern wie Constantin Titel-Petrescu, Gheorghe Cristescu, Al. Constantinescu und D. Pop den Auftrag, in den Reihen der jungen deutschen und österreichischen Offiziere - er hat hier ehemalige Bekannte aus seiner Wiener Schulzeit wieder getroffen -, Antikriegspropaganda zu betreiben. Unter dem Einfluß der bolschewistischen Revolution in Rußland verstärken auch die rumänischen Sozialisten ihre Tätigkeit: Im März 1918 werden in der Druckerei Bræniøteanu in Bukarest Tausende von Exemplaren eines illegalen von Valeriu Marcu verfaßten Manifests gedruckt, in welchem zum offenen Kampf gegen die Besatzer aufgerufen wurde ${ }^{15}$. Die 
Behörden reagierten mit Haussuchungen und Verhaftungen. Von einem Spitzel verraten, wurde auch Marcu verhaftet, in einem Kellerraum der Militärkommandantur gesperrt und vor ein deutsches Kriegsgericht gestellt. Es scheint, daß öffentliche Proteste $^{16}$ sowie die Beziehungen des Ingenieurs Marcu in der AEG-Zentrale in Berlin, sogar zu Walter Rathenau selbst, dazu beigetragen haben, daß das Urteil - die Höchststrafe wäre nicht auszuschließen gewesen - milde ausfiel: Valeriu Marcu wurde zur Deportation nach Deutschland verurteilt, zwei seiner Mitstreiter zu je vier Monaten Gefängnis. Ein Militärzug führte den Verurteilten nach Deutschland, wo er in einem Straflager bei Rastatt interniert wurde.

5 Die folgenden Jahre stellen den Höhepunkt einer Entwicklung dar, die Valeriu Marcu später als "meine Parteinahme für Revolution" bezeichnete. Wir treffen ihn an den verschiedensten Schauplätzen an, immer dort, wo es besonders "heiß" zuging. Nach dem Waffenstillstand vom November 1918 reist er in die Schweiz, um mit seinen Eltern zusammenzukommen: Es war ihr letzter Versuch, ihn $\mathrm{zu}$ einem Abschluß seiner Studien zu bewegen. Doch schon zu Beginn des Jahres 1919 ist er, wie mehrere Quellen zu berichten wissen ${ }^{17}$, in Ungarn, wo er an der Seite Belà Kuns kämpft. Er wird Mitglied der KPD und gleichzeitig Vertreter der rumänischen sozialistischen Jugend bei der Kommunistischen Jugend-Internationale, die 1919 in Berlin gegründet wurde ${ }^{18}$. Seine Erfahrungen aus der Zürcher Zeit sowie die persönlichen Verbindungen zu führenden Bolschewiki haben zur Folge, daß man den jungen Valeriu Marcu mit immer heikleren Aufgaben beauftragt: So nimmt er z. B. im November 1919 an der geheimen Konferenz des Komintern-Sekretariats für Westeuropa in Frankfurt am Main zusammen mit Clara Zetkin, Y.S. Reich, Sylvia Pankhurst, Karl Frank und Mieczyslaw Bronski ${ }^{19}$ teil. Im Januar 1920 ist er zusammen mit dem Komintern-Beauftragten für Deutschland Karl Radek in Berlin-Moabit inhaftiert, wo dieser ihm ein Interview gewährt, das einige Monate später in der Bukarester Zeitung Socialismul erscheint ${ }^{20}$. In derselben Zeitung werden im März 1920 Marcus Berichte aus Mailand veröffentlicht ${ }^{21}$, eindringliche finanzielle Hilferufe an seine Familie in Rumänien bezeugen seine vorübergehenden Aufenthalte in Wien. Unter den deutschen kommunistischen Führern fühlt er sich am meisten Paul Levi verbunden, der damals noch Parteivorsitzender war ${ }^{22}$, dessen Konflikt mit den Vertretern der Komintern sich jedoch schon abzuzeichnen beginnt. Zum Bruch kam es im März 1921, als Levi der Komintern öffentlich vorwarf, sich auf grobe Weise in die Angelegenheiten der KPD einzumischen. $\mathrm{Zu}$ denjenigen, die damals auf der Seite Levis standen, gehörte Valeriu Marcu; er setzte sich auch für die Gründung einer neuen Partei (Kommunistische Arbeitergemeinschaft) ein. Seine Situation innerhalb der Führungsspitze der Kommunistischen Jugendinternationale wurde unsicher: Auf dem 2. Kongreß der Internationale in Jena im April 1921 griff er die Tendenz der Komintern an, die Parteipolitik von außen zu steuern ${ }^{23}$. Es scheint, daß er im selben Jahr auch seine berühmte Reise nach Sowjetrußland unternimmt, vielleicht als Teilnehmer an der zweiten Phase desselben Kongresses in Moskau, sicherlich aber einer persönlichen Einladung Lenins Folge leistend, den er bei dieser Gelegenheit wiedersieht; auch trifft er sich mit Trotzki und Rakovski ${ }^{24}$. Auch Willi Münzenberg, der Sekretär der Internationale, versucht - trotz des Druckes, den einige Funktionäre auf ihn ausüben - noch eine Zeitlang mit Valeriu Marcu zusammenzuarbeiten. Bis Juni 1921 zeichnet Marcu Aufsätze in der Zeitschrift JugendInternationale ${ }^{25}$, obwohl er damals schon in Paul Levis Zeitschrift Unser Weg ${ }^{26}$ veröffentlicht, und im Verlag Junge Garde, einem Sprachrohr der Internationale, erscheint mit Münzenbergs Vorwort eine Broschüre mit dem Titel Die weiße und die rote 
Armee $^{27}$. Auf die ausdrückliche Forderung des damaligen Sekretärs des Exekutivkomitees der Internationale Alfred Kurella wurde Valeriu Marcu doch endgültig im Sommer 1921 aus der Organisation ausgeschlossen ${ }^{28}$.

6 Das ist gewiß ein äußerst schwieriger Moment im Leben des jungen Kommunisten Valeriu Marcu. Er hat keinen "bürgerlichen” Beruf erlernt, ist mittellos, die Genossen von gestern haben sich von ihm abgewendet. Er versteht, daß ihm eine einzige Möglichkeit bleibt, seinen Lebensunterhalt zu bestreiten: das Schreiben. Er liest viel, schafft sich Bücher an, reist - 1922, in den Tagen, als Mussolinis "Marsch nach Rom" stattfindet, hält er sich in Italien auf -, erwägt eine Rückkehr nach Rumänien ${ }^{29}$. Eine wirkliche Unterstützung findet er nur bei Paul Levi und den Freunden aus dessen Kreis: Fritz Schönherr, Bern Meyer und Josef Bornstein sowie bei Willi Münzenberg, der damals schon seinen von der KPD relativ unabhängigen Presse- und Verlagskonzern gegründet hatte. In einer von Münzenberg dirigierten Zeitschrift Sichel und Hammer veröffentlichte Valeriu $\mathrm{Marcu}^{30}$ bis die Komintern erneut intervenierte, um ihn beiseite $\mathrm{zu}$ drängen ${ }^{31}$. Im ebenfalls $\mathrm{zu}$ Münzenbergs Konzern gehörenden Neuen Deutschen Verlag erscheint ein mit dem Pseudonym Gracchus gezeichneter Essay Imperialismus und Friede. Raubkrieg und Revolution und 1925 kommen in der Verlagsreihe "Redner der Revolution" die von ihm edierten Reden Robespierres und Saint-Justs heraus $^{32}$. Von 1924 an arbeitet er regelmäßig für Paul Levis Zeitschrift Sozialistische Politik und Wirtschaft ${ }^{33}$, nach 1925 schließt er sich dem Kreis um Willy Haas und der Literarischen Welt ${ }^{34}$ an, 1926 erscheint sein Name zum ersten Mal in zwei bekannten und verbreiteten Organen der linken Intellektuellen: Die Weltbühne ${ }^{35}$ und Das Tagebuch ${ }^{36}$, aber auch in anderen, wie Roland, Omnibus, Die neue Bücherschau, Das blaue Heft. Seine publizistischen Arbeiten umfassen einen weitgespannten Bogen: Er veröffentlicht Artikel und Feuilletons zu tagespolitischen Themen, kleine historische Abhandlungen, Porträts bekannter Politiker und Ideologen (Sinowjew, Briand, Caillaux, Maurras, Radek, Rakovski), literaturkritische Essays, Buchrezensionen. Die Radikalität seiner frühen Jahre ist etwas abgeschwächt; seinen linksorientierten Überzeugungen, die mit den eigenen Erlebnissen und der alltäglichen Realität der Komintern-Taktik kollidiert sind, bleibt er jedoch treu. Mit der allmählichen Stabilisierung seiner Situation im publizistischen Leben Berlins wird seine Existenz "bürgerlicher", er rückt von der Bohème $a b^{37}$. Marcu frequentiert die literarischen Kreise, gehört zu den Gästen Samuel Fischers $^{38}$ und Ernst Rowohlts ${ }^{39}$, verfaßt Beiträge für die großen liberalen Tageszeitungen (Vossische Zeitung, Berliner Tageblatt), wird Mitarbeiter der Neuen Rundschau ${ }^{40}$, wo viele Größen jener Zeit veröffentlichten, verlangt und erhält die Naturalisierung als "Preuße". 1930 wirft ihm Willi Münzenberg vor, er sei "ein bürgerlicher Schriftsteller geworden, den die Kritiken der Blätter der Grossbourgeoisie ebendo entzücken, wie er sie früher mit uns verspottete" ${ }^{41}$. In der Tat werden seine beiden Porträt- und Essaysammlungen Schatten der Geschichte $e^{42}$ und Männer und Mächte der Gegenwart ${ }^{43}$ sowie seine Monographien Lenin. 30 Jahre Rußland ${ }^{44}$ und Das große Kommando Scharnhorsts ${ }^{45}$, in der zweiten Hälfte der 20-er Jahre erschienen, vom Publikum und von der Kritik besonders gut aufgenommen ${ }^{46}$; einige von den genannten Titeln sowie seine Studie Die Geburt der Nationen ${ }^{47}$ wurden nach ihrem Erscheinen in englischer, französischer, italienischer Übersetzung verlegt ${ }^{48}$.

7 Es beginnt sich jedoch eine große Gefahr abzuzeichnen: der Aufstieg Hitlers, den Marcu schon 1923, nach dem Münchener Putsch als "düstere Perspektive einer kommenden, ungeheuren, blutigen Zeit" voraussieht ${ }^{49}$. Nach der NSDAP-Wahlniederlage im Jahre 1928 gehörte er zu denjenigen, die am wenigsten optimistisch waren. Er warnte vor 
politischer Kurzsichtigkeit und vor der Spaltung der potentiell antifaschistischen Kräfte $^{50}$. Zu jener Zeit, vor allem nach dem Freitod seines Mentors Paul Levii ${ }^{51}$, schwindet Marcus Vertrauen in die Fähigkeit der Arbeiterparteien, dem Ansturm des Nationalsozialismus standzuhalten, immer mehr. Es festigt sich seine Überzeugung, daß eine Lösung nur im Lager der Konservativen zu finden sei, das ihm homogener erscheint und stabiler für eine Konfrontation mit dem Rechtsradikalismus. Symptomatisch sind in diesem Sinne seine immer engeren Beziehungen zu Ernst Jünger, Ernst Niekisch, zum General von Seeckt, dem ehemaligen Chef der Reichswehr, zu den katholischen Politikern Brüning und Treviranus. In der Atmosphäre der bunt zusammengewürfelten Gesellschaften bei Arnolt Bronnen oder anläßlich von Zusammenkünften in seinem eigenen Haus am Wannsee, wo er nach seiner Heirat mit Eva Gerson, der Tochter eines jüdischen Kaufmanns, wohnte, machte sich Verunsicherung breit, wie auch das Bewußtsein, die katastrophale Entwicklung nicht aufhalten zu können ${ }^{52}$. Ernst Jünger erinnert sich an die Worte Marcus, die dieser während eines Spaziergangs zwischen den Villen von Berlin-Dahlem äußerte: "Wissen möcht ich, wer in zehn Jahren darin wohnt. Andere als heut bestimmt" ${ }^{\text {53. }}$.

Bald nach der Machtergreifung durch Hitler beschließt das Ehepaar Marcu, Deutschland zu verlassen. Nach einem kurzen Aufenthalt in Österreich lassen sich die Marcus in Südfrankreich nieder, in einem Ort neben der spanischen Grenze ${ }^{54}$. Die Antwort der deutschen Behörden läßt nicht lange auf sich warten: Marcus gesamte Bibliothek, einige zehntausend Bände, wurde einfach beschlagnahmt. Der Briefwechsel mit Ernst Jünger vermerkt diese Episode mit dramatischem Hintergrund, die erst nach zahlreichen Interventionen der in Berlin verbliebenen einflußreichen Freunde mit der Rückerstattung eines Großteils der Sammlung ihr Ende fand ${ }^{55}$. "Das Leben ratenweise zu verlieren, ist ein feiger Zustand. Aber was ist Feigheit und was Hoffnung?" Für den Emigranten Valeriu Marcu bleibt allein die Hoffnung in einem von Sorgen geprägten Alltag der finanziellen Not, der Enttäuschung und der Unsicherheit. "Durch die Tatsache, daß ich nicht in Deutschland bin, bin ich zu 3/4 besiegt", schreibt er am 24. Januar 1936 an Ernst Jünger, fügt jedoch hinzu: "ich hoffe aber noch während meiner relativen Jugend mit Ihnen Nächte zu zerreden. Ich las noch 1932: Man erlebt alles und das Gegenteil davon".

9 Ein Argument taucht bei Valeriu Marcu immer wieder auf: "Das Schönste, was Deutschland geboren hat, ist und bleibt die 'Autonomie des Geistes'. Diese Autonomie hat eine dynamische Kraft, an die Fichte, Hegel und Clausewitz geglaubt haben, und an die ich auch glaube" ${ }^{56}$. Dieser Glaube sah sich den Nachrichten aus Deutschland gegenübergestellt, die Marcu auf verschiedenen Wegen zu erlangen wußte. Jedes kleine Zeichen von Unzufriedenheit und Widerstand, sorgfältig vermerkt im Briefwechsel mit Gottfried Treviranus, wird, wenn auch nur zeitweilig, zu einem Quell der Zuversicht. "Der wirkliche Dynamismus wird ja erst beginnen. Und der Dynamismus wird sich [...] gegen Hitler wenden. Die Änderung in Deutschland wird kommen, so wahr es ein Deutschland gibt [...]. Die Änderung wird von sozialen Momenten kommen, die sich wieder mit irgend einem Humanismus binden werden. Hitler ist ein ausgesprochenes Provisorium" 57 . Die Verbindungen Marcus zu diversen oppositionellen Gruppen und Grüppchen, die im Land geblieben waren, scheinen seine politischen Voraussagen wenigstens vorläufig - $\mathrm{zu}$ bestätigen ${ }^{58}$. Andererseits engagiert sich Marcu für die antihitleristische Kampagne der Opposition im Ausland; zusammen mit Gottfried Treviranus plant er die Herausgabe einer "konservativen" und zugleich "eminent revolutionären" Zeitschrift ${ }^{59}$; er veröffentlicht regelmäßig in Leopold Schwarzschilds 
Zeitschrift Das Neue Tagebuch ${ }^{60}$; er gehört zu den Gründungsmitgliedern des Bunds Freie Presse und Literatur an, zusammen mit Alfred Döblin, Klaus Mann, Leopold Schwarzschild, Hermann Kesten, Walter Mehring, Josef Bornstein; er ist in der deutschen Sektion des PEN-Clubs aktiv ${ }^{61}$; vom Widerstand der spanischen Republik gegen den Ansturm des Faschismus beeindruckt - "Spanien ist [...] die interessanteste Erscheinung im Jahrzehnt. Es ist der Prolog zu einem Weltstück"62, plant er 1937 eine Spanienreise und eine Zusammenkunft mit dem republikanischen Außenminister Alvarez del $\mathrm{Vayo}^{63}$. Ermüdend und auf die Dauer schädlich sind hingegen die ewigen Konflikte und Zerwürfnisse innerhalb der Emigration. Die Gründe sind manchmal kleinlich - wie im Fall der vorübergehenden Distanzierung von Joseph Roth ${ }^{64}$ oder des Bruchs mit Alfred Neumann -, in anderen Fällen jedoch liegen dem Konflikt fundamentale politische Differenzen zugrunde. Valeriu Marcu gehört zu jenen, die die Taktik und Strategie der Komintern im Kampf gegen den Faschismus offen in Frage stellt. Die Moskauer Schauprozesse, denen Bekannte aus Marcus Jugendzeit, Bolschewiki der ersten Stunde, zum Opfer gefallen sind, dann der Pakt RibbentropMolotow bestärkten ihn in seiner Weigerung, mit den Verfechtern einer breitangelegten "Volksfront" der Emigranten zusammenzuarbeiten ${ }^{65}$. Eine Annäherung an Willi Münzenberg beispielweise, in dessen Zeitschrift Die Zukunft er zu veröffentlichen beginnt, erfolgt erst, nachdem dieser von der Komintern desavouiert worden ist ${ }^{66}$. Die Atmosphäre ist gespannt, und selbst dauernde Freundschaften - wie jene mit René Schickele, Heinrich Mann, Walter Hasenclever, André Gide, André Malraux - können das bedrückende Gefühl der Isolation, unter dem Valeriu Marcu leidet, nicht außer Kraft setzen. Die Briefe, die aus jener Zeit erhalten sind, gewähren einen Blick auf die Folgen jenes Gemütszustandes, dessen Einfluß auf die literarische Tätigkeit unmittelbar ist: "Die Fronten der Kaffeehaustische haben sich hier verändert [...]", schreibt Marcu am 27. September 1940 an Hermann Kesten. "Es sind Anekdoten aus dem Irrenhaus" ${ }^{67}$.

10 Schon ein knappes Jahr nach seiner Abreise aus Deutschland zeichnet Valeriu Marcu drei bedeutende historische Aufsätze zur Bismarck-Ära in Klaus Manns Exil-Zeitschrift Die Sammlung ${ }^{68}$, im Jahrbuch des Amsterdamer Verlages Allert de Lange ${ }^{69}$ (neben Bertolt Brecht, Joseph Roth, Max Brod, René Schickele, Egon Erwin Kisch, Alfred Polg ${ }^{70}$ ar, Alfred Neumann u.a.) und in der Bukarester Revista Fundafliilor Regale $e^{71} .1934$ erscheint sein Buch Die Vertreibung der Juden aus Spanien ${ }^{72}$, das sich eines großen Publikumserfolgs erfreut, vor allem durch die Übersetzungen, die der Reihe nach in den USA, England, den Niederlanden und Argentinien erscheinen - wobei er ironisch-verbittert feststellt: "Da der Autor keine Leser hat, denn das Publikum ist zu Hause geblieben und nur die Rezensenten sind ausgewandert, vermag er sich den Luxus zu erlauben, für eine ideale, d.h. imaginäre Gemeinde zu schreiben"73. Drei Jahre später bringt Allert de Lange das letzte Buch von Valeriu Marcu heraus: Machiavelli. Die Schule der Macht $t^{74}$, eine Biographie, an der er lange Zeit gearbeitet hat, unter anderem auch in Italien. Ein Essay über die militärische Kunst von der Antike bis zur Neuzeit, für den er das gesamte Material zusammengetragen hat und der schon angekündigt ist, bleibt nur Projekt, ebenso kann er eine geplante, autobiographisch gefärbte "Geschichte" der europäischen Arbeiterbewegung, Die Legende des Proletariats ${ }^{75}$, nicht fertigstellen. Es sind überhaupt schlechte Zeiten für die schriftstellerische Produktivität. "Ich versuche, trotz der Ereignisse zu arbeiten, werde aber geschüttelt, wenn ich den Radiokasten öffne" 76 , schreibt er an Treviranus. Südfrankreich war ein zu unsicherer Aufenthaltsort geworden; schon 1939 erwägt Marcu einen Umzug nach England ${ }^{77}$, ein Jahr später 
beginnt er den Papierkrieg zur Erlangung eines US-Visums. Nach dem militärischen Zusammenbruch Frankreichs und der Einrichtung der Regierung Pétain in Vichy hat sich die Lage der deutschen Emigranten drastisch verschlechtert. Trotz der Anstrengungen, die Hermann Kesten in den USA unternimmt ${ }^{78}$, war das Visum noch immer nicht da. Von der allgemeinen Panik erfaßt, unternimmt auch Valeriu Marcu verzweifelte Versuche zur Lösung seiner Probleme; er verschafft sich einen honduranischen $\mathrm{Pa}^{79}$ und sucht sogar die Unterstützung rumänischer Diplomaten ${ }^{80}$. Erst am 15. November 1940 wird die Familie Marcu vom amerikanischen Konsulat verständigt, daß ihre Einreise in die USA bewilligt ist. Obwohl diesmal die französischen Behörden Schwierigkeiten machen, kommt es am 9. Februar 1941 zur überstürzten Abreise aus Frankreich. Die gesamte Bibliothek wird Marcus (und zugleich André Gides ${ }^{81}$ ) Freundin Jane Simone Bussy anvertraut. Der Schwiegervater und der Schwager des Schriftstellers bleiben in Nizza zurück - beide sind umgekommen, der erste in den Gaskammern eines KZ, der zweite durch Freitod. Valeriu Marcu, seine Frau Eva und ihre Tochter Monica verlassen den europäischen Kontinent über Lissabon am 29. März $1941^{82}$.

11 Valeriu Marcu läßt sich in New York nieder, wo er sich total entwurzelt fühlt. Es kostet ihn gewaltige Anstrengungen, alte Kontakte wieder anzuknüpfen, vor allem aber mit den Verlegern zu verhandeln. Von den alten Freunden helfen ihm Heinrich Mann ${ }^{83}$, Hermann Kesten, Fritz Landshoff, Josef Bornstein. Aus Mexico City fordert ihn Egon Erwin Kisch auf, an einer geplanten Emigrantenzeitschrift mitzuarbeiten ${ }^{84}$. Stufenweise wird den Zugang zu den amerikanischen Medien gesucht und gefunden: Aufsätze über den Weltkrieg und zur Lage in Europa erscheinen in American Mercury, Liberty, Commonweal. und die Familie in Rumänien verfolgt seine Kommentare in den deutschsprachigen Sendungen von Voice of America ${ }^{85}$. Parallel dazu arbeitet er an den Manuskripten, die er von Nizza mitgebracht hat. Am 4. Dezember 1942 stirbt Valeriu Marcu in der New Yorker Wohnung von Gottfried Treviranus, während er einigen Freunden ein kurz vorher abgeschlossenes Kapitel über eine Seeschlacht in der Antike vorliest, an einem Herzinfarkt ${ }^{86}$.

Valeriu Marcus stürmische Existenz, die von Brüchen nicht verschont blieb, sein Lebensweg, der oft verworren verlief und von einem dreifachen Exil ohne Rückkehr geprägt war, kann von seinem Werk nicht getrennt werden. Der Person des Autors war, wie ihn verschiedene Zeitgenossen beschreiben, eine verführerische Ausstrahlung eigen: Marcu war leidenschaftlich bis zur Vehemenz im Einsatz für eine Idee, dabei ein Zyniker; ein Bonvivant, der für seine Liebesaffären bekannt war, und zugleich ein fanatischer Bücherfreund, ein glänzender Weltmann, der in den Berliner Salons immer gern gesehen wurde, und ein geborener Tribun. Sein geheimes Vorbild, mit dessen Biographie er sich gründlich auseinandergesetzt hat, scheint Machiavelli gewesen zu sein - jener Mann, der sich lange in der Nähe der Mächtigsten seines Jahrhunderts aufhielt und der, ungeachtet seines komplexen Charakters, die Kunst der Machtausübung hervorragend verkörperte. Mit diesen Gedanken spielt schon einer von Marcus Rezensenten des Machiavelli-Buches, Rudolf Olden, indem er feststellt, daß "eine seltene Verwandtschaft zwischen Held und Autor besteht" ${ }^{87}$. Marcu selber, von der möglichen Analogie eingenommen, nährt diesen Gedanken durch mehr oder weniger deutliche Anspielungen und Fakten, die ihn seiner Meinung nach dem 
italienischen Renaissance-Helden annäherten, sowohl auf der Ebene des Geistes als auch auf jener des Schicksals. Jenseits von komödiantenhaften Allüren, von der Effekthascherei, gegen die auch so mancher Zeitgenosse Marcus nicht gefeit war, bleiben einige unzweifelhafte, wesentliche Eigenschaften bestehen, die der Mensch Valeriu Marcu auf sein Werk übertragen hat: die außerordentliche Gründlichkeit dokumentarischer Investigation, die Fähigkeit, von der Analyse zur Synthese vorzudringen, die innere Freiheit von jeglichen Vorurteilen, eine solide Schule der Dialektik, die er sich im Studium der Werke von Hegel, Marx und Lenin erworben hat, die prophetisch $\mathrm{zu}$ nennende Weitsicht seiner Urteile, die von der späteren Entwicklung in den meisten Fällen bestätigt wird. Dazu kommen die Phantasie und der Skeptizismus, die Ironie und der moralische Anspruch, die Neigung zur Metapher und der spielerische, wache Geist, die Vorliebe für Paradoxa und schockierende Gegensätze, eine provokative Haltung, die gegen alle Klischees gerichtet ist.

Eine präzisere gattungsmäßige "Einordnung" seines Werkes, das sich jedoch den gängigen Etikettierungen entzieht, müßte vorgenommen werden. Seine großen Biographien (über Lenin, Scharnhorst, Machiavelli), die "Geschichte" der Vertreibung der Juden aus Spanien, die "europäischen Profile" (in Schatten der Geschichte und Männer und Mächte der Gegenwart) können, trotz stilistischer und struktureller "Signale" des Textes, nicht als Literatur im engeren Sinne, d.h. Belletristik, angesprochen werden, da das Element der Fiktion fehlt; doch ebenso sind Die Geburt der Nationen oder Die Sozialistengesetze im kaiserlichen Deutschland nicht als Geschichtsstudien im eigentlichen Wortsinn zu betrachten, da die Narration, von allen formalen Zwängen befreit, das Dokumentarische aufsaugt und jeden Anspruch auf Systematik zunichte macht. In der literaturgeschichtlichen Perspektive stellt diese Art von "Prosa der offenen Form" 88 keinen Einzelfall dar. Bei Marcu liegen ihre Wurzeln in der Tradition des Feuilletons, denn er war in erster Linie Journalist, der seine Arbeit den Erfordernissen des Mediums Presse anpaßte und seine Bedingtheit als Zeitungsschreiber reflektierte ${ }^{89}$. Aufschlußreich ist der Kommentar von Willy Haas zu einem seiner "essayistischen" Sammelbände: "Ganz formvollendet ist er in der Erzählung des Gesehenen, Erlebten, dessen, was ihn persönlich affiziert hat. Deswegen eben nenne ich ihn einen Journalisten, und zwar einen von der besten Art"90. Valeriu Marcu äußerte öffentlich seine Verachtung für eine "Literatur", deren soziales Engagement rein theoretischer und deshalb dogmatischer Natur blieb, und für die "Literaten", die jene Literatur produzierten: "Verschlossen in der Stube ihrer abergläubischen Sicherheit, verzichteten sie darauf, selbständig die Mannigfaltigkeit der gesellschaftlichen Veränderungen $\mathrm{zu}$ sehen. Sie sind stumme Beter vor dem großen Drama der Weltgeschichte, und die fruchtbarste Periode der wirklichen Veränderungen ist die unfruchtbarste in der zustimmenden, begeisterten, sich selbst überschreienden, ohne wirklichen Inhalt dahinsiechenden Literatur" ${ }^{91}$. Im Gegensatz zu jenen schätzt er Jack London, den Romancier, der nur über das schrieb, was er aus eigener Anschauung kannte ${ }^{92}$, oder Kurt Tucholsky, dessen Artikel ("Ein Sieg über die Maschine, ein Triumph der Qualität", so Marcu) ${ }^{93}$ ihre eigenen Grenzen überschreiten und dennoch zu Literatur werden. Einige Sätze über Bernard Shaw (dem, neben Anatole France und Heinrich Mann, seine ganze Bewunderung gilt) scheinen Marcus eigenes Programm auszudrücken: "Die Klarheit seiner geschichtlichen Darstellungen wurde schon oft zu einem Argument gegen ihn verwendet. Man wirft dem Historiker und dem Schriftsteller Oberflächlichkeit vor, man behauptet, die Weltereignisse dienten ihm nur zur Fabrikation einiger für die Lachmuskeln und für den Kassenerfolg konstruierter 
Komödien [...]. Der leichte, elegante, flüssige Stil soll eine oberflächliche Gesinnung verraten! Die Männer dieser Argumentation finden nur dann 'Tiefe', wenn sie sich bei der Lektüre langweilen und wenn die von ihnen verehrten Literaten das Geheimnis einiger Auserwählter bleiben" 94 . Es wurde Valeriu Marcu des öfteren vorgeworfen, daß "seine Historie [...] ein Mosaik aus Akten, Porträts und Reflektionen", daß “durch den ständigen Wechsel der Perspektiven [...] jede Bild-Illusion in dem die Details zum Schein einer Einheit zusammengehen könnten, ausgeschlossen" sei ${ }^{95}$, während weniger wohlwollende Rezensenten sich nicht scheuten, der von Marcu vertretenen "feuilletonistischen Geschichtsauffassung" die bewußte Manipulation des Sensationellen vorzuhalten ${ }^{96}$. Gerade die Lesbarkeit seiner Bücher, die keinesfalls mit gewissen "kulinaristischen" Rezepten zu verwechseln war, sollte aber laut anderer der Grund ihres Publikumserfolgs sein: durch den "großartigen stilistischen Schwung" und das "gallische Pathos", die Axel Eggebrecht zu schätzen wußte ${ }^{97}$, durch die geschickte Handhabung sämtlicher Modalitäten der literarischen Prosa (auktoriales und personales Erzählen, Kommentar, Beschreibung, innerer Monolog, erlebte Rede usw.). Im Unterschied zu Emil Ludwig oder André Maurois gelang es Marcu, eine bezeichnende Distanz zum "Objekt" seiner Investigationen zu bewahren; er kannte genau die Grenze, jenseits derer die Geschichte entwertet wird und in billige Bewunderung der zum "Helden" gewordenen Persönlichkeit umschlägt. Die plastische, an Jakob Burckhardts Schriften geschulte Ausdrucksweise ${ }^{98}$, verbindet sich in Marcus Texten mit einer Ironie, die an Anatole France gemahnt ("ein Spott voller Bonhomie, der Spott des Lateiners, der eine kindliche, fast rührende Zärtlichkeit verrät") ${ }^{99}$, und vor allem mit einem ausdrücklichen Skeptizismus, der die intensive Lektüre von David Hume, Leopold von Ranke und Hans Delbrück verrät - eine kritische Haltung, die manchmal in die Nähe dessen rückt, was Joseph Roth "geselligen Zynismus" nannte ${ }^{100}$, und die, im Falle des Lenin-Buches beispielsweise, zu Irritationen und heftigen Reaktionen in KPD-nahen Intellektuellenkreisen Anlaß gab ${ }^{101}$. Diese "skeptische" Art, Geschichte zu schreiben, prägte Marcus Stil - der manchmal zum Aphoristischen tendierte, oder, im Gegenteil, mit Bildern und Metaphern überladen war -, in nicht geringem Maß. Ludwig Marcuse warnte: "Es wäre tragisch und ein großer Verlust, wenn ein Schriftsteller von Marcus Talent, Fleiß und Wissen nicht sehen würde, daß die Skepsis immer nur ein wichtiger Durchgang ist zu überskeptischen Fundamenten. Dem absoluten Skeptiker bleibt nichts übrig als zu schweigen; das wußten schon die Griechen"102.

14 In der Selbstanzeige der Männer und Mächte der Gegenwart bekennt Valeriu Marcu freimütig: "Ich bin zwar kein Historiker", gibt aber den Historikern den Rat, "zwischen zwei dicken Büchern über die Vergangenheit ein dünnes über die Gegenwart zu schreiben"103. Diese Faszination der erlebten Gegenwart - "denn noch nie hat eine Zeit in zwei Jahrzehnten [...] das durchgemacht wie die unsrige" - stellt laut Marcu den einzigen Schlüssel zum Verständnis der Vergangenheit dar, "die beste Schule für die Geschichte". Es gilt für ihn auch der umgekehrte Satz: Zweifellos bietet die Geschichte den Schlüssel zur Interpretation und zum Verständnis der gegenwärtigen Ereignisse. Genauso wie "heute ein Buch über Politik im Sinne Machiavellis, was Tiefe und Qualität anbetrifft, das Gegebene" wäre, so wäre auch ein Buch über Machiavelli und seine Zeit außerordentlich lehrreich. Marcu liefert den Beweis, indem er das Buch schreibt. Denn "vor, während und nach Machiavelli" weisen die Gesetze der Geschichte und die Regeln der Politik frappierende Ähnlichkeiten auf. "Die Menschheit kennt nur eine der Religion ebenbürtige Kraft - die Macht!" ${ }^{104}$, eine brutale und gleichzeitig verführerische 
Kraft. Ihn, den "keuschen Liebhaber der Macht", deren Nähe er sucht wie einst Machiavelli - "nicht um mitzuherrschen, sondern um mitzusehen"105, fesseln die Mechanismen der Macht in all ihren Äußerungsformen, das fundamentale Verhältnis zwischen Individuum und Masse, zwischen Freiheit und Diktatur, zwischen Reaktion und Revolution. Eigentlich kann man seine zwei letzten Bücher, Die Vertreibung der Juden aus Spanien und Machiavelli auch als Meditationen über das Exil-Dasein lesen; der Bezug auf die Gegenwart und sogar auf die eigene Existenz ist unverkennbar. In seinen Betrachtungen verweilt er vor allem bei den Wendepunkten der modernen Geschichte, die für die spätere Entwicklung entscheidend sind, wobei er, vom Phänomen der Macht und ihrer spezifischen Instrumente - Bürokratie, Propaganda und Werbung, Massenhysterie, religiöse Gerichte, Arbeiterbewegung, Taktik und Strategie der Kriegsführung, militärische Organisation - ausgehend, sein Interesse gleichzeitig den machtausübenden Persönlichkeiten zuwendet: "Niemand empfindet diese Notwendigkeit der Veränderung mehr als führende Individuen. Sie wachsen in einer eigenartigen Atmosphäre kommender Dinge auf, werden von der Härte der Welt angezogen [...]. Der Einfluß, den sie erlangen, durch den Völker und Klassen denken und handeln, ist der Macht-Urtrieb. Diese Allgewaltigen, deren Wille zur Wirkung von allen Schrecken der Hölle nicht vernichtet werden konnte, sind zugleich die Allerschwächsten, abhängiger von der Notwendigkeit als gewöhnliche Sterbliche in ihren Tafel- und Ehefreuden" ${ }^{106}$. Die Reflexion über die Macht umfaßt auch die Analyse der gegenseitigen Beziehungen zwischen jener und den Intellektuellen, einschließlich über das eigene Verhältnis zur Macht. Als Biograph beobachtet Valeriu Marcu die "Mächtigen" als Elemente eines umfassenden, ununterbrochen wirkenden Prozesses der geschichtlichen Entwicklung mit ihren Gesetzmäßigkeiten und Zufällen. Die Perspektive dieser Entwicklung beschränkt sich nicht auf die Gegenwart, sondern umreißt auch - jenseits aller Utopie - ein mögliches Bild der Zukunft. Diesen Prozeß nennt Marcu "hart, kalt, gleichgültig, folgerichtig, unbarmherzig"; "es sind Kräfte, die ringen, Interessen, die morden, gigantische Anstrengungen, die sich messen, Willensballungen, die sich vor dem dunklen Hintergrund des geschichtlichen Horizonts in blitzender Aktion entladen"107. Daher auch seine Abneigung gegen das Jonglieren mit jeglicher Propaganda ${ }^{108}$, seine Verachtung für Schriftsteller, die nichts als Worte "einer vorgeschriebenen Meinung" 109 wiederholen. Dabei stellt er immer wieder zwei Grundhaltungen des Intellektuellen zur Macht einander gegenüber: Auf der einen Seite steht der begeisterte "Optimismus", feige und verlogen, auf der anderen das freimütige Bekenntnis zur Vernunft: "Deshalb sind seine Bücher [von Ignazio Silone] wichtiger, bedeutender, aufklärender als alle offiziellen Staatsakte aller dieser Wachsfiguren der Politik, die täglich einen Status quo paraphieren und beschwören, der in den Tiefen der Nationen nirgends existiert"110. Gerade Marcus eigene "Sozialisation" ist symptomatisch für den inneren Werdegang eines Menschen, der die Skepsis der kritischen Beobachtung jeglichem Aktivismus vorzog. "Wenn ein Jüngling mit den neugierigen Augen eines Zwanzigjährigen die Welt betrachtet, verliebt er sich in Prinzipien", schrieb Valeriu Marcu in dem kurzen Vorwort zu Schatten der Geschichte. "Er glaubt, aus Grundsätzen alles ableiten, durch sie allein alles verstehen zu können [...]. In dieser Rebellion steckt mehr Optimismus als Überhebung. Bald jedoch beginnen schmerzliche Prozesse, die ertragen werden müssen, um die Selbständigkeit, die Autonomie des eigenen Denkens zu erlangen". Diese neue Eigenschaft, die er "ohne zu trauern oder [sich] zu freuen" erlangt hat, wird in seinem Werk wichtiger als zeitweilige Kontakte und dezidiert politische Haltungen. Die Lektüre seines 
unbeendeten Manuskriptes zur Legende des Proletariats gibt zu erkennen, was dieses Buch werden konnte: ein Zeugnis des Irrwegs eines Einzelnen, der sich mühsam vom kollektivistischen Mythos der Menschheitserlösung befreit, um im skeptischen Alleingang das eigene individuelle Dasein behaupten zu dürfen, und zugleich eine durchaus nüchterne Betrachtung des im 20. Jahrhundert in apokalyptischer Dimension wiederholten menschlichen Wahnsinns. Die jugendliche Identifizierung mit der Marxschen Fortschritts-Utopie, die sehr früh ihre unerbittlich menschenfeindlichen Mechanismen offenbarte, wird in den zustandegebrachten Kapiteln dieser Memoiren mit der Weisheit desjenigen geschildert, der in den späten 30-er Jahren seine schlimmsten Visionen der totalitären Macht in dem NS-Terror in Deutschland und in dem Sowjet-Terror in Rußland verwirklicht sah. Die frühe Reife seines politischen Denkens weist auf eine "prise de conscience" hin, die Valeriu Marcu zusammen mit einem Victor Serge oder Panait Istrati zu einer der wichtigsten Gestalten der früh ernüchterten nachrevolutionären "Renegaten" qualifizierte.

Das Ende der Illusion von einer aufgeklärten, intelligenten, wirksamen, der Ration untergeordneten Macht fällt für Marcu mit der realen Katastrophe Deutschlands unter Hitler zusammen. "Sie [die Nazis] sind so erfüllt von einer permanenten 'Nacht der langen Messer', so getrieben von ihrer Gefühlsprimitivität, daß sie nicht einmal als reservatio mentalis eine allgemeine Idee der Erlösung haben. Die Gewalt nach innen und nach außen ist ihnen die Bildnerin der Welt" ${ }^{111}$. Einst war Valeriu Marcu von der einsamen Geste eines Benedetto Croce beeindruckt, der sich im Senat des faschistischen Italien gegen die "Ordnung" Mussolinis aussprach; Ein Kopf ist mehr als dreihundert Kehlköpfe - so betitelte er den Artikel, in dem er Croce ein kleines Denkmal, als Beispiel für einen offenen, unverkrampften Widerstand des Geistes, setzte ${ }^{112}$. Acht Jahre später schrieb er Ernst Jünger aus Süd-Frankreich, daß "ein Kopf [...] gleich 1000000 Kehlköpfe" ${ }^{113}$ sei. Als der Emigrant sein Buch über Spaniens Juden im 15. Jahrhundert zu schreiben begann, um "gegenwärtige Angelegenheiten" zur Sprache zu bringen ${ }^{114}$, schien es, als ob er schon eine Ahnung hätte, von dem, was kommen sollte. "Der Antisemitismus ist [...] eine ideale Übung zum Massenzynismus" ${ }_{115}$. Die Freisetzung solcher Kräfte spielt dann eine entscheidende Rolle in der allen rationellen Entwürfen zuwiderlaufenden Gestaltung absolutistischer und schließlich diktatorischer Macht: "die Übergänge zwischen Ja und Nein, zwischen Freundschaft und Feindschaft, das Spiel der eigenen Seele, der Prozess, wie 'Dingsda' zum Führer und Vorbild wird, wie das Selbstgefühl der Unterordnung wirkt, wie die innere Hingabe an die Diktatur entsteht, wie die Gruppe auf den einzelnen drückt, wie aus Sklaverei Gehorsam und Hingabe wird, wie der Knecht im Menschen seine eigenen Züge im Idol da oben erkennt, und wie sich im dunklen Gewirr der Empfindung die Furcht mit der Nützlichkeit, mit dem Egoismus, mit der Angst um das tägliche Brot verbindet" ${ }^{116}$. Die Gegenwart leuchtet unter dem Bannstrahl des obssesiven déjà-vu-Erlebnisses auf, wirft ihre Schlaglichter auf die Vergangenheit, Hitlers Deutschland und Torquemadas Spanien spiegeln sich gegenseitig; im Mittelpunkt steht das memento als Warnung an all jene, die als freie Menschen der Verführung der sogenannten "freiwilligen Knechtschaft" 117 zum Opfer fallen. Der bürokratischen Maschinerie des Staatsterrors, die die Inquisition inthronisiert hatte, ist es gelungen, Spanien für mehr als drei Jahrhunderte in "das ruhigste Land Europas" ${ }^{118}$ zu verwandeln - eine widernatürliche, versteinerte Ruhe, die rückblickend die absolute negative Utopie in nuce verkörpert: "Der Mensch muß auf Millimeter genau funktionieren und parieren; er hat keine andere Seele, keine andere Funktion als ein Kolben, als eine Schraube, als eine Schiene. 
Jede Art von Freiheit ist nur eine Ausrede für Dummheit, Faulheit, Schlauheit, Spießbürgerei oder Perversität. In jedem Begehren nach Freiheit manifestiert sich nur die Unordnung überwundener Zustände. Freiheit ist nichts anderes als Maschinenoder Bilderstürmerei" ${ }^{119}$. Es ist die für Valeriu Marcu in der spanischen Erfahrung offenbarte Orwellsche Landschaft, die im Maßstab des 20. Jahrhunderts von den vom Hitlerismus und Stalinismus patentierten Modellen vervielfältigt wurde.

“Nous vivons dans l'attente d'événements formidables; et parfois l'un de nous s'écrie: exactement ce qu'avait prédit Marcu", schrieb André Gide am 27. Juli 1941 aus Nizza an Valeriu Marcu, der seit kurzem in New York eingetroffen war ${ }^{120}$. Die Bemerkung Gides bestätigt Marcus Ruf als klarsichtigen Beobachter der eigenen Epoche, die Anerkennung der prophetischen Dimension seiner Warnrufe vor Resignation, Schwäche oder Aussöhnung mit den Diktatoren. Ein Fragment aus einem im Februar 1939 veröffentlichten Text nimmt symptomatisch den Ablauf des 2. Weltkriegs vorweg: "Übergewaltige Koalitionen gegen das Dritte Reich müssen entstehen, weil der Nationalsozialismus alle Wunden der Welt reizt, weil er nicht nur alle bestehenden Landesgrenzen bedroht, sondern auch jeden einzelnen Menschen innerhalb dieser Grenzen. Die Energiematerie des Individuums, seine Selbsterkenntnis und seine Selbstbeherrschung werden alles Wollen und jedes Ziel gegen das Dritte Reich richten. In jedem Land werden die übrigen politischen Parteiungen verschwinden, und nur eine Frage wird übrigbleiben und täglich lebendiger werden: Widerstand oder kein Widerstand gegen die allbedrohende Berchtesgadener Gewalt. Freilich kann dieser politische, erst in letzter Linie militärischer Prozeß möglicherweise jahrelang dauern. Die Bildung eines gewaltigen kriegerischen Willens ist ein Vorgang, der vielleicht in jedem Lande durch eine Reihe von Krisen, Niederlagen, Revolutionen und Gegenrevolutionen hindurch muß. Während dieses Prozesses werden vielleicht morsche Staats- und Parteigebilde verschwinden. Aber das und vieles andere, was sich auf der politischen Weltbühne bläht, ist sekundär. Primär ist nur die Endwucht in einem schier ungeahnten Kampf der Sterblichen gegen Deutschland. Die alte Mutter Erde wird in unendlicher Schlachtenglut flammen"121.

17 Es wurde Valeriu Marcu nicht gegönnt, das siegreiche Ende der Völkerschlacht zu erleben.

\section{NOTES}

1. In der von Klaus Mann und Hermann Kesten herausgegebenen Anthologie demokratischer Schriftsteller Europas Heart of Europe, New York 1943 wird Marcu zusammen mit den französischsprachigen Autoren Martha Bibesco und Panait Istrati als "rumänischer" Schriftsteller designiert, wobei diese willkürliche Einordnung dadurch zu erklären ist, daß die von der wenig übersetzten und deswegen auch kaum bekannten rumänischen Literatur nichts ahnenden Herausgeber unbedingt alle Nationalliteraturen Europas in ihrem Buch repräsentiert haben wollten. Vgl. auch Klaus Manns Brief an Kesten in Klaus Mann, Briefe und Antworten, München 1975, Bd. 2, S.20. 
2. Vgl. Andrei Corbea-Hoisie, Valeriu Marcu, in Deutschsprachige Exilliteratur seit 1933, Bd.4: Bibliographien. Schriftsteller, Publizisten und Literaturwissenschaftler in den USA, hrsg.von John M. Spalek, Konrad Feilchenfeldt und Sandra H. Hawrylchak, Bern/München 1994, S.1248-1259.

3. Die spärliche Präsenz Valeriu Marcus in den deutschsprachigen Literaturlexika ist in diesem Sinne symptomatisch. Er wird jedoch im Band 12 von Kindlers Literatur-Lexikon, Darmstadt 1964, durch einen Artikel von Peter Glaser (S.11043) und im Band 7 vom Literatur-Lexikon. Autoren und Werke deutscher Sprache, hrsg. von Walther Killy, Gütersloh/München durch einen Artikel von Heiner Widdig (S.481), sowie in einigen anderen wichtigen Nachschlagwerken: W. Sternfeld/ E.Tiedemannn, Deutsche Exil-Literatur. Eine Bibliographie, Heidelberg 1970; Liselotte Maas, Handbuch der deutschen Exilpresse, München 1976; Biographisches Handbuch der deutschsprachigen Emigration nach 1933, Bd.2, München/New York/London/Paris 1983, vermerkt.

4. Vgl. die verschiedenen Beiträge zum Thema "Exil und Remigration" in "Exilforschung. Ein Internationales Jahrbuch", Bd. 9 (1991), hrsg. von Claus-Dieter Krohn.

5. Ernst Jünger, Strahlungen II, in Werke, Bd.3.Stuttgart 1960, SS 443-444, 455-459. Marcu diente Jünger auch als Modell für die Gestalt Siggi Jersons in seinem Roman Aladins Problem, Stuttgart 1983.

6. Vgl. die Reprints von Valeriu Marcus Exilbücher Die Vertreibung der Juden aus Spanien (1991) und Machiavelli. Die Schule der Macht (1993) bei Matthes \& Seitz München, die ein beträchtliches Presseecho hatten (siehe auch Andrei Corbea, Memoiren rund um Valeriu Marcus Memoiren, in "Neue Literatur" 2 (1995), S.86-91); in dem Literatur-Jahrbuch des Verlags Der Pfahl wurden ebenfalls mehrmals Texte von Marcu oder über Marcu veröffentlicht, darunter die Korrespondenz mit Fritz Brupbacher, Gottfried Treviranus, André Gide, Heinrich Mann und Ernst Jünger, hrsg.von Andrei Corbea, in Der Pfahl, V (1991), S.92-127.

7. Vgl. Marcus Essay Leo Trotzki oder die Dreieinigkeit der Philosophie, des Krieges und der Politik, in Schatten der Geschichte, Leipzig 1929.

8. Christian Rakovski, in "Das Neue Tagebuch" (NTB) 13 (1938).

9. Willi Münzenberg, Die dritte Front, Berlin 1930, S 223.

10. Die Pariser Blutwoche und Die Jugendbewegung in Rumänien, in "Jugend-Internationale" (JI) 4 (1916).

11. Lenin in Zürich, in "Foreign Affairs" 3 (1943).

12. Die Informationen stammen aus einem sich im Josef Bornstein-Archiv des Leo Baeck-Instituts New York befindenden Manuskript von Valeriu Marcu, von dem wir das entsprechende Fragment in "Neue Literatur" 2 (1995) veröffentlicht haben.

13. In JI 7 (1917).

14. In JI 10 (1917).

15. Siehe im Archiv des ZK der RKP, Dokumentarbestand Nr. 28, Mappe Nr. 5 und 118; vgl. auch Ecaterina Arbore-Ralli, Die sozialistische Bewegung in Rumänien, in "Kommunistische Internationale" 7-8 (1919).

16. Ein Manifest, reproduziert in Documente din istoria miøccerii muncitoreøti sin România 1916-1918, Bucureøti 1958, S.100, fordert ausdrücklich die Freilassung von Marcu.

17. Vgl. Ernst Jünger, Strahlungen II, Stuttgart 1960, S. 444 und Heinrich Brüning, Briefe und Gespräche, Stuttgart 1974.

18. Vgl. B. Lazich, M. Drachkowich, Biographical Dictionary of the Comintern, Stanford 1973, S. 258.

19. B. Lazich, M. Drachkowich, Lenin and the Comintern I, Stanford 1972, S. 177.

20. De vorbo cu Radek, in "Socialismul2 71 (1920).

21. Ebd. 59, 63, 70 (1920).

22. Vgl. Curt Geyer, Die revolutionäre Illusion, Stuttgart 1976 und Karl Retzlaw, Spartacus, Frankfurt am Main 1976.

23. R.Schüller, A.Kurella, R.Chitanow, Geschichte der kommunistischen Jugend-Internationale, Berlin 1929-1931, Bd. 2, S. 164, 168. 
24. Mehrere Quellen, unter anderen sein Trotzki-Essay aus dem Band Schatten der Geschichte und sein sich im New Yorker Leo Baeck Institut befindendes Manuskript bestätigen dieses Datum der Reise.

25. Der letzte Aufsatz in JI 10 (1921) mit dem Titel Neue Form des bürgerlichen Militarismus ist mit dem Pseudonym Wallmar gezeichnet.

26. In der Nr. 1 (1921) erscheint Marcus Aufsatz Zur Krise der Kommunistischen Internationale. (Die Zeitschrift nannte sich bis zur Nr. 6 "Sowjet").

27. Wallmar, Die weiße und die rote Armee, St. Petersburg/Berlin 1921 (Internationale JugendBibliothek Nr. 18).

28. Babette Gross, Willi Münzenberg, Stuttgart 1968, S. 119.

29. Die rumänische Gesandtschaft in Berlin soll ihm den Paß aus politischen Gründen verweigert haben.

30. Sein erster Aufsatz erschien in der Nr. 6 (1923).

31. Babette Gross, a.a.O., S. 161.

32. Maximilien Robespierre, Reden, NDV, Berlin 1924 und Reden von Antoine de Saint-Just, NDV, Berlin 1925.

33. Unter dem Pseudnym V.M.Huber beginnt er seine Mitarbeit in der Nr. 48 (1924) mit dem Pamphlet Traktat über die Glückseligkeit eines Volkes, eine Verfassung wie die Weimarer sein eigen zu nennen; eine frühere Mitarbeit unter uns unbekannten Pseudonymen ist nicht auszuschließen.

34. In der Nr. 2 (1925) erscheint sein Aufsatz Lenin als Stilist.

35. Er debütiert mit dem antifaschistischen Pamphlet Die alten und die neuen Römer, Reprint $1926 \mathrm{I}$, S.960.

36. In der Nr. 2 (1926) veröffentlicht er den Artikel Prinz Carol of Rumänien.

37. Siehe auch Max Krell, Das alles gab es einmal, Frankfurt am Main 1961, S. 185 und Axel Eggebrecht, Der halbe Weg, Reinbek 1975, S. 186.

38. Oskar Loerke, Tagebücher, Heidelberg 1976, S.187. Mehrere Quellen glauben zu wissen, daß Leo Trotzki durch Marcus Vermittlung im S. Fischer Verlag zu veröffentlichen begann.

39. Ludwig Marcuse, Mein 20. Jahrhundert, München 1960, S. 147.

40. Er debütiert 1927 mit dem Essay Jean Paul Marat und Antoine de Rivarol.

41. Willi Münzenberg, a.a.O., S. 224.

42. Die erste Ausgabe bei Hoffmann \& Campe, Berlin/Hamburg 1926; die zweite bei Paul List, Leipzig 1929.

43. G. Kiepenheuer, Berlin 1930.

44. Paul List, Leipzig 1927.

45. Paul List, Leipzig 1928.

46. Lobende Rezensionen erschienen in "Weltbühne", "Die literarische Welt", "Die Literatur", "Das Tagebuch", "Sozialistische Politik und Wirtschaft", "Die Gesellschaft", "Die schöne Literatur", "Preußische Jahrbücher", "Die Neue Rundschau”, "Querschnitt”, usw.

47. S. Fischer, Berlin 1931.

48. Bei wichtigen Verlagen, darunter MacMillan, Viking Press, Payot, Mondadori usw.

49. Babette Groß, a.a.O., S. 161; siehe Marcus Aufsatz Die Revolution und der Faschismus, in "Sichel und Hammer", Nr. 7 (1923).

50. Karl Retzlaw, a.a.O., S. 284.

51. Vgl. Charlotte Beradt, Paul Levi, Frankfurt am Main 1969.

52. Ernst Jünger, a.a.O., S. 457.

53. Ebd., S. 459.

54. Die erste französische Anschrift der Familie Marcu lautete: Etablissement du Parc, La Franqui-Plage, Par Leucate, Aude.

55. Siehe Anmerkung 6.

56. Brief von 17. Dezember 1933 an Ernst Jünger. 
57. Brief von 17. Dezember 1938 an Gottfried Treviranus.

58. Er berichtet Treviranus mehrfach von solchen Verbindungen, darunter auch von Ernst Jünger gesandte Emissäre.

59. Brief von 10. Juli 1837 an Treviranus.

60. Sein erster Artikel Ein Ketzer unter Sozialisten erscheint in der Nr. 16 (1937).

61. Vgl. den Katalog der Ausstellung Der deutsche PEN-Club im Exil, Deutsche Bibliothek Frankfurt am Main, S. 254).

62. Brief vom 28. Januar 1937 an Jünger.

63. Brief vom 7. Mai 1937 an Treviranus.

64. Joseph Roth, Briefe, Köln 1970, S. 447, 449.

65. Marcus Verhältnis zu einem Teil der Emigration muß aus der Perspektive der prinzipiellen politischen Differenzen betrachtet werden, die ihn schon vor 1933 von den linken Gruppierungen trennten. Im NTB nimmt Valeriu Marcu des öfteren gegen die stalinistischen Ausschreitungen Stellung - siehe die Artikel: Tuchatschewski , 26 (1937); Die Propaganda, 28 (1937); Stalins Galgen und Trotzkis Bücher, 8 (1939); Geist und Gestalt des Kommunismus, 17 (1940) u.a.

66. Gottfried Treviranus, Für Deutschland im Exil, Düsseldorf 1973, S. 73; vgl. auch Marcus Brief vom 1. Mai 1939 an Treviranus. Margarete Buber-Neumann (in Von Potsdam nach Moskau, Stuttgart 1957, S. 457) kann sich nicht erklären, wieso Münzenberg, als er sich 1939 in Todesgefahr befand, nicht wenigstens für eine Zeit bei Valeriu Marcu in Nizza Unterschlumpf suchte.

67. Hermann Kesten, Deutsche Literatur im Exil, Wien 1964, S. 151.

68. Die Sozialistengesetze im kaiserlichen Deutschland, Nr. 19 (1935).

69. Die Kräfte und Widersprüche deutscher Einheit, S. 81.

70. 1871 (in rumänischer Sprache), Nr. 2 (1934).

71. Querido Verlag, Amsterdam.

72. Schon 1937 in einer in Oslo erschienenen Studie von Odd Eidem (Diktere i Landflyktighed) wird Marcus Buch als eines der ersten typischsten Werke der deutschen Exilliteratur charakterisiert.

73. Bücher, die ich schreiben möchte, in NTB, Nr. 48 (1937).

74. Das Buch wurde übersetzt in den USA, Finnland, Polen und Argentinien.

75. Skizzen, Projekte und Fragmente aus den Manuskripten befinden sich im Bornstein-Nachlaß im New Yorker Leo Baeck Institut.

76. Brief vom 26. März 1939.

77. Ebd.

78. Siehe Kestens Brief vom 31. August 1940 an American Guild, aufbewahrt bei der Deutschen Bibliothek Frankfurt am Main.

79. Hans Albert Walter, Deutsche Exilliteratur, Darmstadt/Neuwied 1972, Bd. 2, S. 29.

80. Die Behauptung, daß Valeriu Marcu sich nach Kriegsausbruch als Rumäne "frei und unbehelligt" bewegen, im Unterschied zu den den deutschen Emigranten, die unter Beobachtung standen und zum Teil auch interniert wurden, wie man es in René Schickele. Leben und Werk in Dokumenten, hrsg. von Fr. Bentmann, Nürnberg 1974, zu lesen bekommt, wurde von Frau Eva Marcu in einem an uns gerichteten Brief nuanciert: obwohl Marcu seit Jahren kein rumänischer Bürger mehr war, hat ihm der rumänische Konsul in Béziers dank der Intervention der von Varian Fry geleiteten Organisation der Rettung von europäischen Intellektuellen einen "echten" rumänischen $\mathrm{Paß}$ ausgestellt.

81. Vgl.André Gide et Dorothy Bussy: Correspondence III (“Cahiers André Gide" 11), édition établie par Jean Lambert et annotations par Richard Tedeschi, Paris 1982.

82. Siehe Marcus Artikel Diary of an escape from Europe, in “American Mercury”, Nr. 11 (1941).

83. Siehe Anmerkung 6.

84. Hermann Kesten, a.a.O., S.219.

85. Die Information stammt von seiner Schwester Angela Giurcaneanu. 
86. Gottfried Treviranus, a.a.O., S. 107.

87. Ein Spezialist der Macht, in NTB, Nr. 5 (1937).

88. Hugo Friedrich, Montaigne, Bern/München 1967, S. 312.

89. Siehe seinen Artikel Presse und Gesinnung, in "Literarische Welt", Nr. 29 (1929).

90. In LW Nr. 4 (1926) unter dem Titel Junger Journalismus.

91. Linker Stammtisch, in LW Nr.37 (1929).

92. Jack London oder der Optimist aus Elend, im Band Schatten der Geschichte.

93. Kurt Tucholsky oder die Grenzen des Journalismus, in LW Nr. 6 (1928).

94. Bernard Shaw unerquickliches Christentum, in LW Nr. 24-25 (1926).

95. Ludwig Marcuse, Skeptische Geschichtsschreibung , in "Das Tagebuch", Nr. 11 (1930).

96. Otto Heller, Ein smarter Blutmystiker, in "Neue Deutsche Blätter", Nr. 10 (1934).

97. Axel Eggebrecht, Ein europäisches Buch, in “Weltbühne” Nr. 22 (1926).

98. Lest Jacob Burckhardt! , in LW Nr. 4 (1926).

99. Siehe Anmerkung 97.

100. In seiner Rezension zu Männer und Mächte der Gegenwart, in LW Nr. 6 (1930).

101. Siehe Kurt Kerstens Kritik in "Die neue Bücherschau”, Nr. 6 (1928).

102. Siehe Anmerkung 95.

103. In "Das Tagebuch", Nr. 10 (1930).

104. Machiavelli, S. 97.

105. Ebd., S. 67.

106. Biographie und Biographien, in Männer und Mächte der Gegenwart, S. 18.

107. Ebd., S. 22.

108. Vgl. den Essay Reklame, oder Abschied von Europa, in Männer und Mächte der Gegenwart.

109. Ignazio Silone, in NTB Nr. 50 (1938).

110. Ebd.

111. Weltrevolution des Nihilismus, in NTB, Nr. 2,3,4 (1938).

112. In LW, Nr. 27 (1929).

113. Brief vom 28. Januar 1937.

114. Brief an Jünger vom 23. August 1933.

115. Siehe Anmerkung 111.

116. Siehe Anmerkung 109.

117. Von der freiwilligen Knechtschaft, in NTB, Nr. 29 (1938).

118. Die Vertreibung der Juden aus Spanien, S. 204.

119. Ebd.

120. Siehe Anmerkung 6.

121. Siehe Anmerkung 111.

\section{RÉSUMÉS}

Der deutsch schreibende rumänische Autor Valeriu Marcu wurde im Jahre 1899 in Bukarest geboren. In seiner Jugend war er ein überzeugter Kommunist, weswegen er das Land wiederholt verlassen mußte und des öfteren verhaftet wurde. 1919 ließ er sich in Berlin nieder, verbrachte aber weiterhin viel Zeit auf Reisen. Ab 1925 publizierte er kulturkritische Artikel und kurze historische Abhandlungen in Zeitschriften wie „Die Weltbühne“ und „Das Tagebuch“. Der 
Putschversuch der Nazis im Jahre 1923 ließ ihn eine düstere Zukunft für Deutschland vorausahnen. In der Folge war Marcu bemüht, sich enger an konservative Kreise um Ernst Jünger und General von Seeckt anzuschließen. Nach der Machtergreifung der Nazis verließ er mit seiner Familie Deutschland, ließ sich in Südfrankreich nieder, bevor er 1941 nach New York auswanderte. Nur wenige Wochen nach seiner Ankunft in den Vereinigten Staaten starb er an einem Herzinfarkt.

Valeriu Marcus literarische Arbeiten sind vor allem Biographien (Lenin, Scharnhorst, Macchiavelli) und historische Abhandlungen (Die Geschichte der Vertreibung der Juden aus Spanien). Es sind Reflexionen über die Liebe zur Macht, die er als einzige ebenbürtige Kraft zur Religion gelten läßt, und über die Existenz als Exilant. In den Biographien zeichnet er die Mächtigen als Element eines ununterbrochen wirkenden geschichtlichen Entwicklungsprozesses, der sich nicht nur auf die Gegenwart, sondern besonders auf die Zukunft bezieht.

L'écrivain roumain d'expression allemande Valeriu Marcu est né en 1899 à Bucarest. Dans sa jeunesse, il était un communiste convaincu, ce qui lui a valu de devoir quitter son pays et de se faire arrêter à plusieurs reprises. Il s'installa à Berlin en 1919, tout en continuant à beaucoup voyager. À partir de 1925 il publia des articles de critique culturelle et de brefs essais historiques dans des revues telles que la ,Weltbühne' et ,Das Tagebuch'. La tentative de putsch des nazis en 1923 lui fit pressentir un avenir sombre pour l'Allemagne. Par la suite Marcu réagit en cherchant à se rapprocher de plus en plus des milieux conservateurs gravitant autour de Ernst Jünger et du Général von Seeckt. Après la prise du pouvoir par Hitler, il quitta, avec sa famille, l'Allemagne, s'installa dans le sud de la France avant d'émigrer en 1941 à New York. Il mourut d'un infarctus peu de temps après son arrivée aux Etats-Unis. Les écrits de Valeriu Marcu sont essentiellement des biographies (Lénine, Scharnhorst, Machiavel) et des essais historiques (L'histoire de l'expulsion des Juifs d'Espagne). Ce sont des réflexions sur l'amour du pouvoir qu'il considère comme la seule force égalant celle de la religion, ainsi que sur l'existence en tant qu'exilé. Dans ses biographies, les puissants de ce monde sont présentés comme élément d'un processus historique en perpétuelle évolution qui a prise non seulement sur le présent mais aussi sur l'avenir.

\section{INDEX}

Mots-clés : Roumanie, émigration roumaine, littérature de la migration

oeuvrecitee Lénine - Scharnhorst - Machiavel - l'histoire de l'expulsion des Juifs d'Espagne

\section{AUTEURS}

ANDREI CORBEA-HOISIE

Jassy/Wien 